Corresponding author Phone +421556022625 E-mail address:marek.vagas@tuke.sk (Marek Vagaš, Ing. PhD.)

Article information Article history: AMS-Volume15-N0.4-00131-1 Received 28 February 2011

Accepted 12 May 2011

\section{Increasing Degree of Automation of Production Systems Based on Intelligent Manipulation}

\author{
Marek Sukop, Mikuláš Hajduk, Vladimír Baláž, Ján Semjon, Marek Vagaš*
}

Department of Production Systems and Robotics, Nëmcovej 32, 04200 Košice, Slovak Republic

\section{BIOGRAPHICAL NOTES}

Mikuláš Hajduk, prof. Ing. PhD., born in December 12, 1950, Malé Ozorovce. He graduates as professor in 1999 at Technical University of Košice in the filed of "production systems with industrial robots and manipulators". Nowadays he is the head at department of production systems and robotics and also an authorized person for robotic and production techniques. He is chairman and also member of board of state examiners. His scientific and research work focuses on the field of research and development of production systems and robotics. An accent is put on the issue of designing and maintenance of automatic robotic systems based on method implementation of computer intelligence.

Vladimír Baláž, Ing. PhD., born in Ocotber 31, 1955 in Prešov. He graduates in 1976 at the Slovak Technical University at Bratislava, Faculty of Electrical engineering and information technology, Department of Automatization with title Ing. In March 2009 he completed a doctorate. This time operates at a Technical university of Košice, Faculty of Mechanical Engineering, Department of Production Systems and Robotics as an Assistant Professor. It addresses the issue of applied research in the field of control and programming robots and assembly and programming of PLC systems.

Jan Semjon, Ing. PhD., born in August 25, 1979 in Snina. He graduates in June 2003 at the Technical University of Košice, Faculty of Mechanical Engineering, Department of Production Systems and Robotics with title Ing. In October 2008 he completed a doctorate. This time operates at a Technical university of Košice, Faculty of Mechanical Engineering, Department of Production Systems and Robotics as an Assistant Professor. It addresses the issue of robotized workplaces for welding and assembly.

Marek Sukop, Ing. PhD., born in Ocotber 2, 1976 in Kosice. He graduates in 2001 at the Technical University of Košice, Faculty of Mechanical Engineering, Department of Production Systems and Robotics with title Ing. In March 2009 he completed a doctorate. This time operates at a Technical university of Košice, Faculty of Mechanical Engineering, Department of Production Systems and Robotics as an Assistant Professor. It addresses the issue of applied research in the field of robosoccer with multiagent system applications.

Marek Vagaš, Ing. PhD., born in Aprilr 16, 1981 in Trebišov. He graduates in 2005 at the Technical University of Košice, Faculty of Mechanical Engineering, Department of Production Systems and Robotics with title Ing. In August 2009 he completed a doctorate. This time operates at a Technical university of Košice, Faculty of Mechanical Engineering, Department of Production Systems and Robotics as research employee. It addresses the issue of applied research in the field of designing of robotics and humanoid applications. 


\section{KEY WORDS}

Production Systems, Intelligent Manipulation, 3D vision, KUKA robot

\begin{abstract}
In project implementation by the Centre for management of technical, environmental and humane risks research for permanent development of production and products in machinery industry, specifically for the activity 3.2. Which deals with automation and robotization of production systems, the main target is increasing degree of automation with focus on intelligent manipulation on 3D basis. This target is being applied by large scale use of robots for various manipulation and technological application. The solution is based on two camera sensors which enable intelligent manipulation on $3 \mathrm{D}$ basis. For this purpose a sensor and software for object recognition have been specified. The solution will be applied and verified on KUKA robot for „pick and place” cases.
\end{abstract}

\section{Introduction}

The development in the field of production system equipment is being transferred from classical sensor components to robots equipped by $3 \mathrm{D}$ camera systems. The main reason is that classic approaches are not sufficient enough to meet new demands with regard to manipulation, picking or certain parameters measuring. At present the camera sensors are an important part of robot equipment for improving its peripheral abilities. Nowadays the research is based on 3D vision as well as creating three-dimensional model by one of the industries which represent the main trend in robotics [1].

In case there are at least two sensors of video signal (camera) available, it is possible to create threedimensional picture of an object. By determining position of particular objects in every signal source and by matching results we will get exact geometrical concept of the object and its properties [9]. Pursuant to that we can transfer reference plane in arbitrary distance in horizontal direction $\mathrm{x}$ and at this place we are able to measure corresponding displacement value (in pixels). By means of the following calculation the exact distance between the sensor and the object will be determined. Under the condition that two of co-ordinal axes will be preserved, e.g. vertical $y$-axis and z-axis.

\section{Methods of 3D vision}

The basic geometrical principle of 3D scanning vision is shown on Fig. 1. It consists from two camera sensors placed side by side. The object before them is captured and digitized. The orientations of captured object are intersecting and their axes of vision are parallel [2]. Distance between camera sensor and target can be calculating through the distance ratio between cameras (b) and reference plane from the camera ( $r$ ) to absolute difference between measured displacement (a) and (c). Then:

Using the images from the camera sensor and

$$
Z=\frac{(b \times r)}{|a-c|}
$$

through the various functions needed for processing is 3D vision of object clearly defined. This data are further processed to control the robot system.

The properties that are essential for recognizing of the image resolution of obtained image, frame rate per seconds and stability information about intensity and colors are taken into considerations. Accordingly, the 3D dimensional vision is distinguished into two ways.

\section{Using of two 2D camera sensors \\ 2. Using of 3D camera}

\subsection{Using of two 2D camera sensors}

To be able to obtain sufficiently precise data it is necessary to calibrate both camera sensors. The internal camera sensors parameters (calibration matrix, coefficients of distortion) and external (mutual position and orientation of both camera sensors) are determinates at calibration [3].

Positions of 3D points are calculating from location of points in both cameras through epipolar geometry. The geometry which is deal with relationships between scanned pictures in both camera sensors is called epipolar geometry.

For obtaining three dimensional position of points it is appropriate to transform the images so that pairs of corresponds points have the same $y$ - coordinate. Then we only need assign selected point that corresponds in second camera sensor line and it is not necessary to search whole image. It is advisable to processed the image before recognition because this procedure of recognition is simpler [10]. Some of the negative characteristics of images obtained from camera sensors (see Figure 2) are also removed during preprocessing. 


\subsection{Using of 3D camera}

The object is scanned at the workplace with 3D camera sensor and image data obtained from the transformation is applied to adjust the image so that it is decomposable [4]. Individual parameters will be searched in distributed status, and also will be determined the characteristics of the subject. Position of points, which indicates the object is obtained after their detection.

Three dimensional locations of points (see Figure 3) can be calculated based on image recognition through industrial 3D camera sensor with two lenses. This information can be used for measuring of dimensions, thickness, position and orientation.

The correction of formed distortion from camera sensor lens and image distortion can be eliminated by measuring the image distortion and than correcting of image. Corrections transformed into Cartesian space and geometrical dependings between object and camera sensor are calculated through calibration. This is very important issue, especially in the field of robotics. Calibration is necessary for every measurement of 3D vision. With comparison of scanned object and CAD model we can accurately determine the position and orientation of any object through 3D vision sensor.

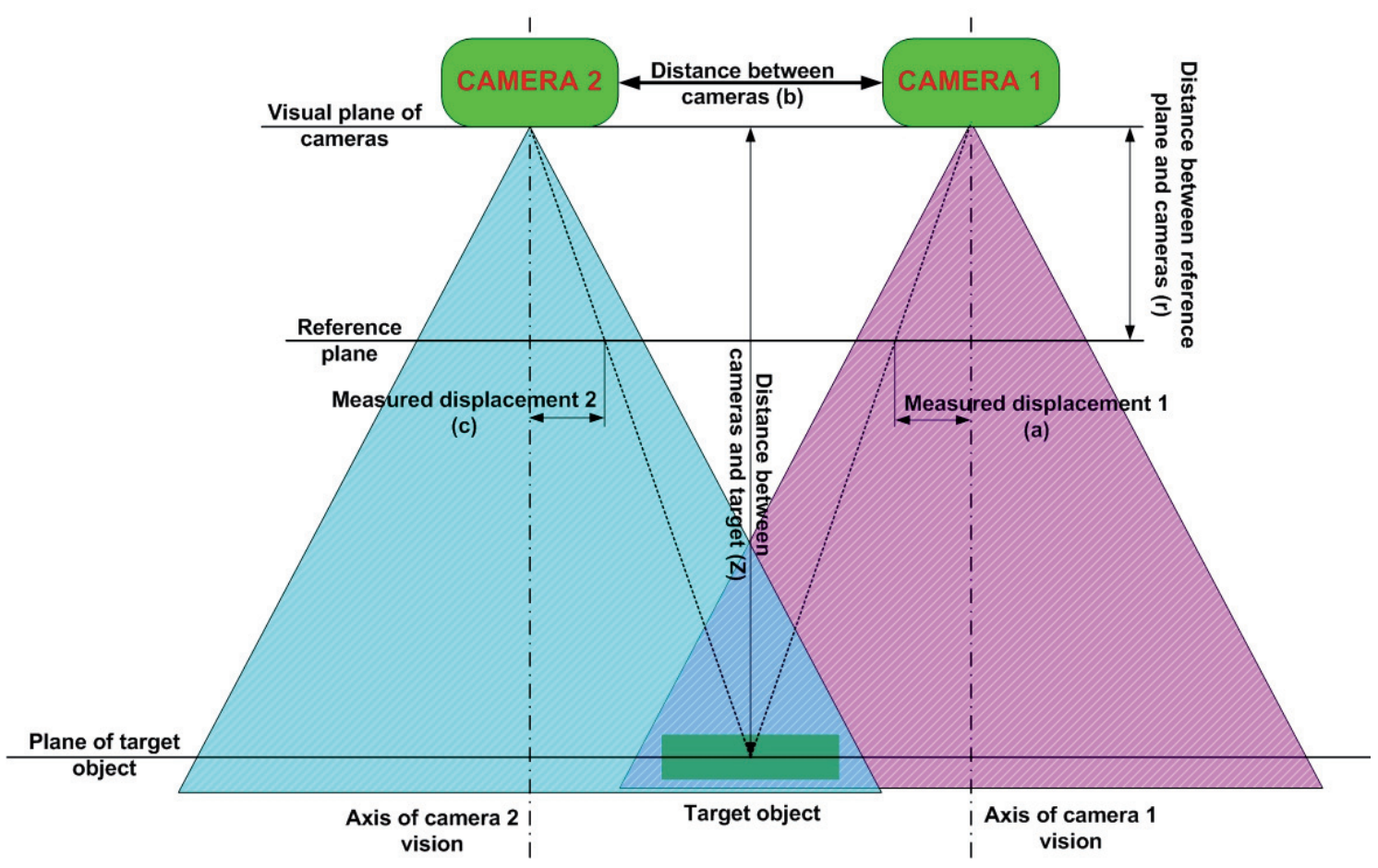

Fig. 1: Geometrical principle of 3D vision by camera sensors.

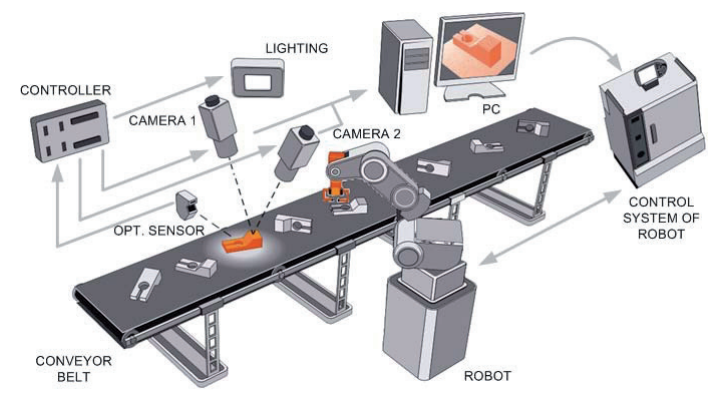

Fig. 2: Application of 3D camera vision by two camera sensors.

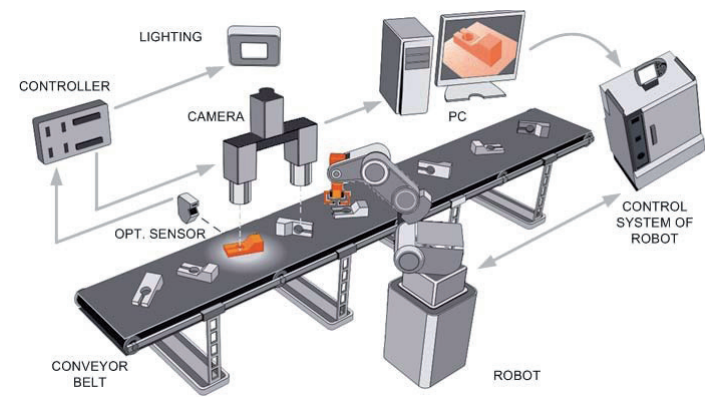

Fig. 3: Application of 3D camera vision by 3D camera sensor. 


\section{Intelligent handling with robot KUKA}

Using a camera sensor mounted directly on the KUKA robot and adjustable light intensity we can functionally works in the full six axes of freedom in 3D. The robot is programmed to place a camera sensor and adapt lighting to the achievement of optimal image capture. Developed software processes the image and sends it via Ethernet directly to the robot control system [5]. This approach to visual processing brings the benefit especially in the smart handling of parts, which eliminates the need of storage the components in a specified position which in the final sum significantly reduces work times.

On Fig. 4 is shown the proposed principle of intelligent manipulation of the parts that are chaotically arranged in a stack of pallets. With the camera sensor is image captured and further evaluated. Image recognition of components is needed mainly to smooth hold with effector and also for handling to the conveyor belt.

Figure 5 shows the principle of intelligent manipulation on the basis of classification of components into the prepared container of pallets. Vision sensor located on the end members of the robot evaluates the movement of parts on conveyor belt in regular intervals. The next step is to determine the spatial coordinates of part, which is located closest to the conveyor and is determined to move to the stack of pallets [6].

Figure 6 shows the classification of different types of components on the conveyor and their storage into the prepared container of pallets. The camera sensor evaluates the image, rotation and position of individual components depending on time. The followed step is picking a particular component and its manipulation with respect to a specific location in the stack of pallets.

The advantages of using 3D vision camera lie in the following areas:

- Lower labor costs (there is no need to use human) - Lower investment costs (eliminates the need for further automation and fixtures)

- Greater flexibility (only one robot even greater variability in production)

- Higher quality (ability to recognize any defective components)

- Greater safety (eliminates the ergonomic requirements of staff, who may be from the prior occupation moved to more productive tasks)

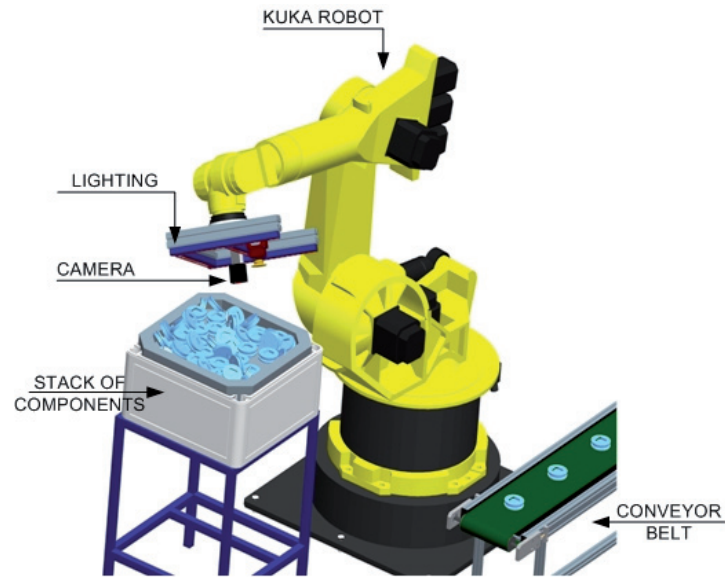

Fig. 4: Intelligent manipulations of chaotically arranged components.

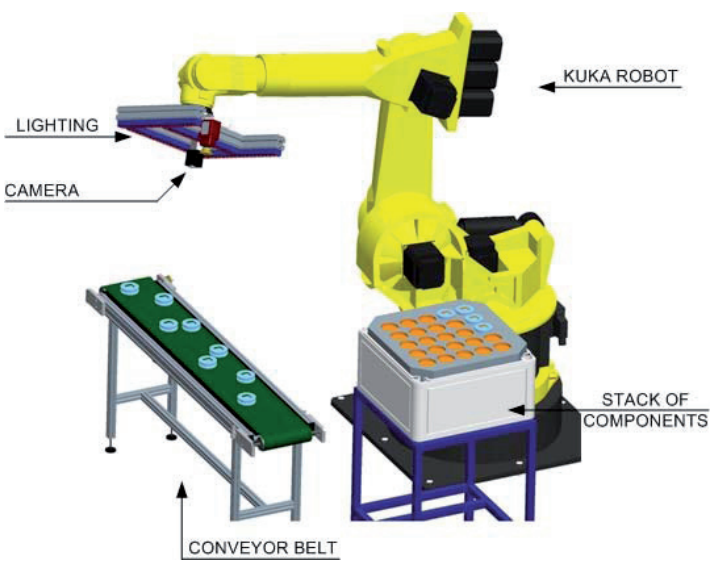

Fig. 5: Intelligent manipulations for insertion into stack of pallets.

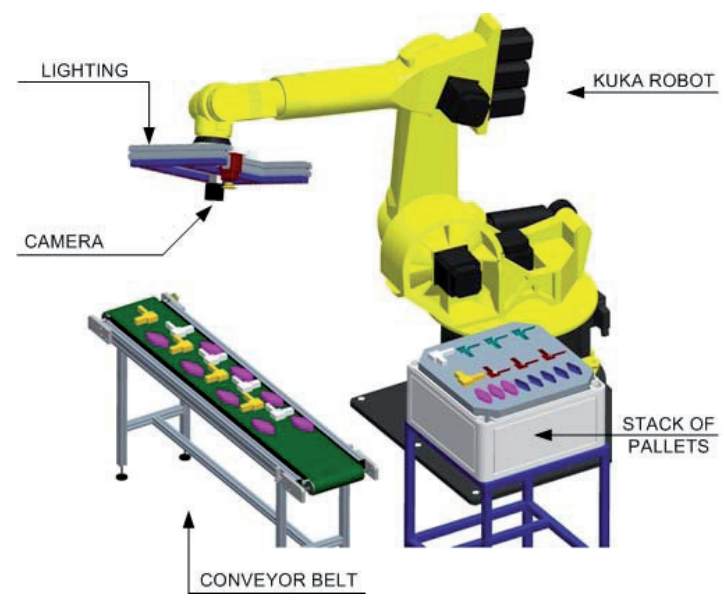

Fig. 6: Intelligent manipulations of different types of components. 
Based on the means of a specific activity of 3.2 were selected following components necessary for $3 \mathrm{D}$ vision.

\section{- F 150 C10E-ST - evaluation unit \\ - $F 150 \mathrm{KP} 2 \mathrm{~m}$ - ST - programming console \\ - F 150 - S1A - ST - camera sensor (without lens) \\ - F 150 - US $3 M$ - ST - connecting cable to camera sensor \\ - F 150 A20 - ST - module for two camera sensors \\ - F 150 VA - ST - wired to the enlargement of mod- ule}

- MM 35 CCTV - camera lens

- Lighting for camera sensors

- Box with accessories

- Software + monitor

\section{Control algorithm}

The principle of decision - making algorithm for griping the parts (shown on Fig. 7) is based on a nearest components that can be grasped [7]. Therefore applies:

IF $\quad \mathrm{N}=1 \rightarrow$ to the components that are closest to the end effector

-

•

$\mathrm{N}=\mathrm{K} \rightarrow$ for component, which is furthest from the end effector; $(1$; number of components in a stack)

$3 \mathrm{D}$ vision algorithm is in terms of managing distributed into two main categories. The first approach is based on the use of the target object image transmission properties from a camera sensor and 3D space is than calculated using the direct method of determining errors of transmitted signal. Signal errors are used as the basis for calculating the control signals which are expression for the control and movement of robot.

The second approach is to control a robot with camera sensor based on calculation of spatial relationships between coordinate system of camera sensor and the target coordinate system of the object [8].

The transfer of information between Kuka robot and 3 D vision system $F 150$

Communication between Kuka robot and F 150 vision system is realized through the channel RS 232C. In this case the serial interface must be configured for communication through CREAD / WRITE by Fig. 8 .

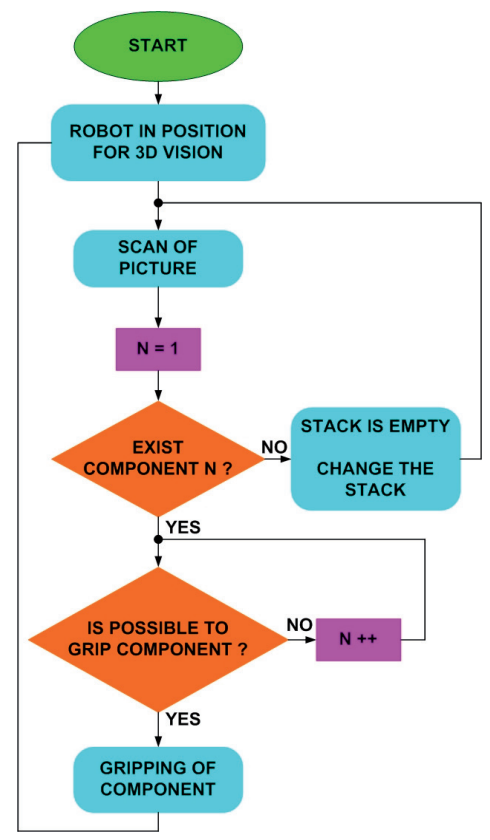

Fig. 7: Decision - making algorithm for gripping of components.

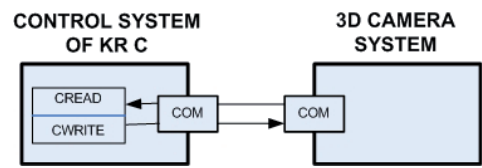

Fig. 8: Communication between Kuka robot and vision system F 150 .

To achieve this goal it is necessary to perform the following steps:

1.assign a serial communication channel to the operating system

2.configure the serial communication channel, where it is necessary to select the transition process 3. configure the transition process

4. perform a cold restart of control systém of a robot

The serial interface must be assigned to the $V x$ Works operating system in order to transfer data were used through CREAD/CWRITE. For KSS 5.4 a 5.5 (Communication system software) is normally assigned to COM3, if KSS 7.0 is it COM2. The self configuration of the serial interface is implemented in the file SERIAL.INI:

[COM3]

BAUD $=9600 ; 110,150,300,600,1200,2400,4800$, $9600,19200,38400,57600$

CHAR_LEN $=8 ; 7,8$

STOP_BIT $=1 ; 1,2$ 
PARITY $=2 ;$ EVEN $=2, \mathrm{ODD}=1, \mathrm{NONE}=0$

$\mathrm{PROC}=1 ; 3964 \mathrm{R}=1, \mathrm{SRVT}=2, \mathrm{WTC}=3, \mathrm{XON} /$

$\mathrm{XOFF}=4$

Protocol 3964R is commonly used for data transfer. In case of difficulties in communication with external devices can be used a simpler protocol XON/ XOFF. Protocol SRVT and WTC serve only for service needs of Kuka robot. Setting the transition protocol

\section{R:}

[3964R]

CHAR_TIMEOUT = 500; msec, max. Interval between two characters

QUITT_TIMEOUT = 500; msec, max. Time of waiting RS robot to character DLE

TRANS_TIMEOUT $=500 ;$ msec,

MAX_TX_BUFFER $=2 ; 1 \ldots 5$, max. Number of output buffers

MAX_RX_BUFFER $=10 ; 1 \ldots 20$, max. Number of input buffers

SIZE_RX_BUFFER $=1 . .2048$; dimensions of the receiving memory in bytes

PROTOCOL_PRIOR $=1 ; \mathrm{HIGH}=1, \mathrm{LOW}=0$, priority

Example for sending data from the robot position:

\section{Extract of main program:}

DEFDAT SEND; Initialization

Declaration; MW_T = \#SYNC

INT HANDLE; Instruction

DECL STATE_T SW_T, SC_T; OPEN_P ()

DECL MODUS_T, MW_T; WRITE ()

ENDDAT;CLOSE_P ()

END

DEF SEND ()

\section{Conclusion}

Implementation of the 3D camera system allows us to obtain three - dimensionally oriented information's about objects and relations between the vision sensor and given object. For the proposal of 3D camera system will be used a control system based on TCP / IP and for data processing, image and calculations will be used a powerful computer. The security of camera system communication and whole control of computer is converted using the control module for Ethernet F150-C10E-2, the camera sensor will be mounted on the end effector of the robot. A specific sensor resolution is $512 \times 484$ pixels with sixteen frames per seconds.

\section{Acknowledge}

This contribution is the result of the project implementation: Center for research of control of technical, environmental and human risks for permanent development of production and products in mechanical engineering (ITMS: 26220120060) supported by the Research \& Development Operational Program funded by the ERDF.

\section{References}

[1] Cheng F., Chen X.: Integration of 3D stereo vision measurements in the industrial robot applications, International Conference, Engineering and Technology, Nashville, 2008 , ISBN 978-1-60643-379-9

[2] Inaba Y., Sakakibara S.: Industrial intelligent robots in Springer handbook of automation 2009, Part C, 349-363, ISBN 9783-540-78831-7

[3] Saedan M., Ang M.: 3D vision based controlon an industrial robot, In: Robotics and application, 2008

[4] Nguyen M.: Vision based intelligent robots, Institute of Measurement Science, SPIE, Vol. II, 2009

[5] Hajduk, M, Semjon, J, Baláž, V, Tuleja, P,:Training Workstation with the KUKA Robot, IN: Optirob 2007: Bren Publishing House, ISBN 9789736486562

[6] Hajduk, M, Baláž, V, Sukop, M, Evin, M, Vagaš, M.: Robotized Cell for Palletization with CCD Camera, IN: Optirob 2007: Bren Publishing House, ISBN 9789736486562

[7] Kováč, M, Babjak, Š.: Integration of Product Innovation Techniques in Automotive Component Design, IN: Acta Mechanica Slovaca, Volume 14 No. 1/2010

[8] Svetlík, J, Demeč, P.: Curved Rotary Module for Modular Construction of Motion Structures, IN: Acta Mechanica Slovaca, Volume 15 No. 1/2011

[9] Evin, E.: Designing of Dual Phase Steel Sheets for Auto Body, IN: Acta Mechanica Slovaca, Volume 15 No. 2/2011

[10] Spišák, E, Badida, M, Kmec, J, Sobotová, L.: Identification of Structure of Hydro-Erosion Operation, IN: Acta Mechanica Slovac, Volume 15 No. 2/2011 